Fecha de recepción: diciembre 2017 Fecha de aceptación: marzo 2018 Versión final: julio 2019

\section{Modelo procedimental para la caracterización y valoración de residuos de aparatos eléctricos y electrónicos, RAEE}

Marcelo E. Venegas Marcel ${ }^{\star}$, Andrés Ricardo Navarro Carreño ${ }^{\star \star}$ y Evelyn Pamela Alfaro Carrasco ${ }^{\star * \star}$

\begin{abstract}
Resumen: El siguiente trabajo surge a partir del relevamiento de información que permitió evaluar la situación actual de los residuos eléctricos y electrónicos (RAEE) en Chile. Identificando los métodos de caracterización en cada material para establecer condiciones, valorizaciones y procedimientos en el manejo adecuado de éstos en post de la nueva Ley de Responsabilidad extendida del Productor (REP). El resultado obtenido establece un modelo procedimental aplicable a la Enseñanza de la Ingeniería y el Diseño entre otras disciplinas, que permitirá sistematizar la toma de decisiones y la planeación desde una perspectiva Ecoeficiente, potenciando de esta forma proyectos de interés tecnológico y económico para el manejo sostenible de los residuos eléctricos y electrónicos.
\end{abstract}

Palabras claves: Ley REP - Residuos Eléctricos y Electrónicos - Gestión de residuos - Método para la caracterización.

[Resúmenes en inglés y portugués en la página 298]

${ }^{(*)}$ Marcelo E. Venegas Marcel. Es Diseñador Industrial Universidad de Valparaíso y Magister en Construcción y Tecnología de la madera Universidad del Bío Bío. Experiencia en el área de la docencia universitaria como en el ejercicio profesional ligado al desarrollo de productos; así como consultor y evaluador para la acreditación de instituciones de educación superior. Orientación acadé-mico-investigativa dentro del área tecnológica de los materiales, para el Diseño, la Manufactura y la Producción con miras a la sustentabilidad medioambiental. Profesor de cátedra en las carreras de Diseño e Ingeniería en: Universidad Federico Santa María, Universidad de Santiago, Universidad de Chile, Universidad Tecnológica Metropolitana.marcelo.venegas@usm.cl

${ }^{(*)}$ Andrés Ricardo Navarro Carreño. Es Ingeniero en Prevención de Riesgos Laborales y Ambientales de la Universidad Técnica Federico Santa María, Licenciado en Gestión Ambiental de la Universidad Católica del Norte, con formación profesional en Sistemas de Gestión Ambiental e Institucionalidad Ambiental. Entre el 2012 y 2015 se desempeña como Docente Carrera de Ingeniería en Seguridad y Prevención de Riesgos de la Universidad Andrés Bello, sede República, Santiago. Desde 2014 a la actualidad como docente de UTFSM, sede Viña del Mar en las carreras de Ing. en Prevención de Riesgos Laborales y Ambientales, Ing. en Fabricación y Diseño Industrial, y Técnico Ambiental.

andres.navarro@usm.cl 
${ }^{(* *)}$ Evelyn Pamela Alfaro Carrasco. Es Ingeniero Civil Químico, Directora del Departamento de Química y Medio Ambiente de la Universidad Técnica Federico Santa María Sede Viña del Mar, representante de la institución en diversas instancias de vinculación con el medio como son el Consejo Consultivo Regional del Medio Ambiente de la Región del Libertador Bernardo O’Higgins, Comisión de Medio Ambiente de la Asociación de Industriales de la V región, Comité para el Cambio Climático de la Región de Valparaíso, Directorio del Centro para el Mejoramiento Genético de Frutales Biofrutales S.A., Directorio del Centro Regional de Estudios para la Alimentación Saludable, entre otros. evelyn. alfaro@usm.cl

\section{Introducción}

Chile se ve expuesto a cambios desde la óptica medioambiental debido a la promulgación de la ley REP 20.920 (responsabilidad extendida al productor, mayo 2016) la que establece una política marco para la gestión de los residuos. Dicha ley, ya en vigencia tendería a posicionar al país en una lógica Económica Circular, que permitiría cerrar el ciclo virtuoso entre productor, recolector e incluso usuario (McDonough \& Braungart, 2005). Ciclo para el que los paradigmas y enfoques de la Enseñanza en general, así como para la Ingeniería y el Diseño Industrial, deben ir adaptándose (UNESCO, 2012).

Un tipo de residuo de crecimiento exponencial, son los de carácter tecnológico (TIC) dentro de los que se encuentran los RAEE que son los provenientes de los productos conocidos como Aparatos Eléctricos y Electrónicos (AEE) que operan gracias a la electricidad o campos magnéticos. Pero son el resultado también de la manufactura y el ensamblaje de un conjunto diverso de estructuras, partes y componentes. Estas mediante el uso intensivo de materias primas poliméricas, metales ferrosos y no ferrosos, sílice y otros compuestos complejos, se convierten en un desafío al intentar desecharlas e integrarlas restaurativa o regenerativamente en un determinado ecosistema (Fundación Ellen MacArthur, 2015). Siendo así una constante preocupación para la Investigación Universitaria y las autoridades ligadas al medioambiente, aun cuando la atención mayoritaria se ha centrado en la elaboración de sistemas de recolección, transporte y disposición final de desechos. La excepción quizás está, en las tecnologías desarrolladas para el manejo y aprovechamiento de placas de circuito impresas, PCB, por el valor de las fracciones de metales preciosos (oro y plata) que las componen (Park Y J \& Fray D J, 2009). Sin embargo, otros componentes como los del tipo polimérico, mantienen aún tasas relativamente bajas en cuanto a su recuperación (Habib K, Parajuly et al, 2015) ignorando, por cierto, la valoración de las propiedades físicas y químicas aún intactas de estos materiales, impidiendo de este modo el advenimiento e implantación completa y efectiva de la llamada Minería Urbana (Fernández F, 2013).

La situación respecto de la gestión de los RAEE en Chile aún es limitada. Mientras en Europa la tasa de reciclaje se acerca al 49\% (UNU, 2017) en nuestro país sólo se eleva al 3\% (Heinz, B. Silva, U Ott D, 2009). No resulta extraño entonces entender la falta de paráme- 
tros, obligaciones y jurisdicción que evidencia la ley REP chilena, que, aunque promulgada en el 2016, en la práctica aún no se encuentra operativa completamente. Esta situación es una evidencia y por tanto una falta de información relevante que permita establecer condiciones para la caracterización y valorización de material, asociable a la gestión para la recuperación (MMA, 2017), por lo que el desarme de los AEE como actividad profesional todavía se torna emergente, concentrándose principalmente en la prestación secundaria de servicios a grandes compañías o bien estableciéndose de modo informal, tratando de obtener ganancias marginales a partir de los residuos generados por los hogares (Heinz, B. Silva, U Ott D, 2009).

Según se desprende de lo dicho, el trabajo que se presenta pretende establecer un esquema procedimental, tendiente a determinar valorizaciones, caracterizaciones y parámetros generales aplicables a la gestión de los RAEE, disponiendo información necesaria ante los requerimientos de formulación de proyectos tecnológicos centrados en la recuperación de partes, componentes y sus materias primas. Por lo que se establecen los siguientes objetivos procedimentales:

- Identificar el procedimiento para la caracterización referido al tipo de material residual obtenido, enunciando propiedades relevantes para el mercado nacional.

- Detallar las características de los residuos valorizables y disposición final de estos.

- Aplicar instrumentos para la clasificación y jerarquización de los RAEE estableciendo condiciones y procedimientos adecuados para su posterior gestión.

\section{Método}

La metodología propuesta considera la elaboración de un modelo de procedimientos ejecutable de manera secuencial (Ver Figura 1), el que en todas sus etapas es aplicado a cinco (5) unidades RAEE analizadas en laboratorio. El criterio para su selección, obedece a la prevalencia de estos residuos en vertedores chilenos (MMA, 2009). 


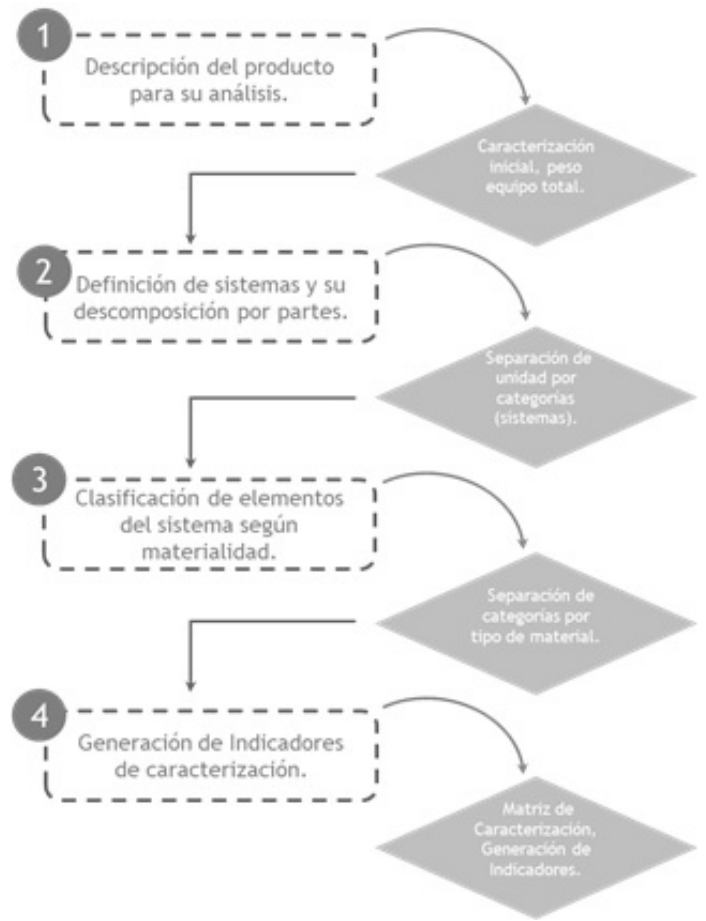

Figura 1. Diagrama general metodología propuesta. Fuente: Elaboración de los autores, según Zeng X et al, 2017.

Las siguientes son como etapas del proceso de caracterización y su detalle:

\section{Descripción funcional del producto para su análisis.}

El activador de todo el proceso corresponde a la selección de la unidad de análisis, de la que se debe declarar una descripción general indicando su función. En esta etapa también se deben registrar datos de entrada, como peso total de la unidad de análisis (Pt) dimensiones, volumen, para su clasificación.

\section{Definición de sistemas y su descomposición por partes.}

Para el inicio del desmantelamiento manual de la unidad de análisis, se definen los sistemas o categorías que corresponderán al primer equipo del estudio, individualizándose según sus características y/o funciones, para posteriormente pesar grupos de componentes según las categorías definidas. Para este estudio se definirán seis (6) categorías para clasificar las partes obtenidas por desmontaje, condicionándose en al menos el 10\% del peso total del equipo para ser considerada como una categoría válida a evaluar (Ver Tabla 1). 


\begin{tabular}{|l|l|l|l|}
\hline ID & \multicolumn{1}{|c|}{$\begin{array}{c}\text { CATEGORÍA } \\
\text { (sistema individual) }\end{array}$} & \multicolumn{1}{|c|}{ DESCRIPCIÓN } & \multicolumn{1}{|c|}{$\begin{array}{c}\text { PESO } \\
\text { (Gramos) }\end{array}$} \\
\hline C1 & Carcasa & $\begin{array}{l}\text { Componente exterior del equipo, obtenidos de la primera separación } \\
\text { de piezas }\end{array}$ & \\
\hline C2 & Fuente de poder & $\begin{array}{l}\text { Grupo de componentes internos que entregan la energización } \\
\text { al sistema, a la vez que conecta con la alimentación de energía } \\
\text { exterior }\end{array}$ & \\
\hline C3 & Estructura & Piezas que constituyen el equipo dando su forma & \\
\hline C4 & Unidad funcional n & $\begin{array}{l}\text { Unidad interna con funciones específicas del equipo, distintas a las } \\
\text { descritas (carcasa, fuente de poder y estructura) }\end{array}$ & \\
\hline C5 & Componentes electrónicos & $\begin{array}{l}\text { Placas de circuito impreso, PCB, módulos electrónicos } \\
\text { (condensador, etc.), cables, conectores. }\end{array}$ & \\
\hline C6 & Otros & Otros materiales no clasificados anteriormente & \\
\hline
\end{tabular}

Tabla 1. Categorías para separación inicial de unidad y análisis. Fuente: Elaboración de los autores.

\section{Clasificación de componentes según materialidad.}

Definidos los sistemas de análisis inicial se separarán todos los componentes de estas categorías según tipología de material, obteniendo valores de peso individual por material identificado. La Tabla 2 presenta tipos de materiales presentes en razón del volumen total del equipo y por tanto el interés en su estudio. Se requiere la clasificación según naturaleza de material e individualización de cada uno de ellos.

\begin{tabular}{|l|l|}
\hline \multicolumn{1}{|c|}{ MATERIAL } & \multicolumn{1}{c|}{ DESCRIPCIÓN } \\
\hline Polímero & ABS, PPE, HIPS \\
\hline Metales & Chatarra de Hierro, Acero, Cobre y/o Aluminio \\
\hline PCB & $\begin{array}{l}\text { Placa de circuito impreso (PCB, Print cuircuit Board), mezcla de basematerial no } \\
\text { conductor más algunos metales }\end{array}$ \\
\hline Vidrio & Tubos de rayos catódicos, o no contaminado \\
\hline Otros & $\begin{array}{l}\text { Mezcla de materiales no clasificados. Ej: cables, conectores, tornillos, pequeñas piezas } \\
\text { de polímeros o metales }\end{array}$ \\
\hline
\end{tabular}

Tabla 2. Tipología de materiales obtenidos de un RAEE. Fuente: Elaboración de los autores.

Para el análisis de los datos obtenidos de unidad caracterizada, los componentes y sus especificaciones se organizan en una matriz que permita un análisis en base a categorías propuestas de componentes y tipología de materiales (Ver Tabla 3). 


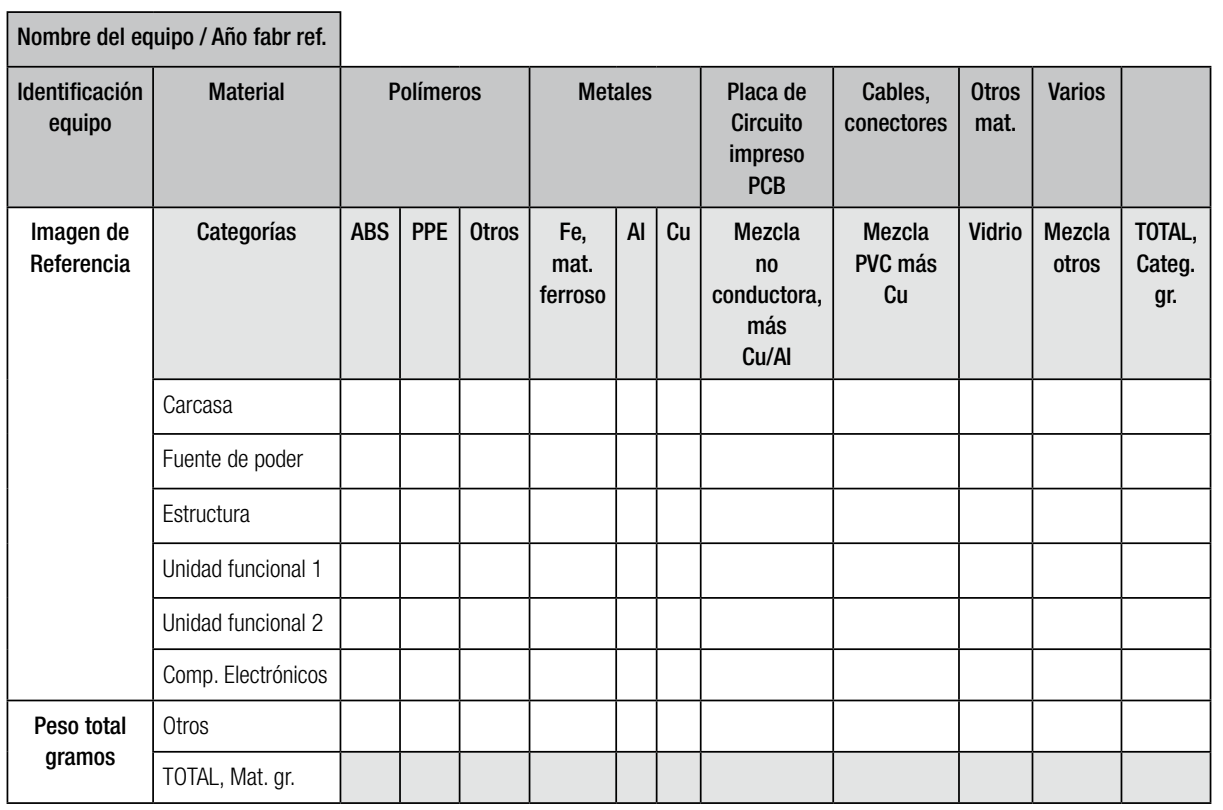

Tabla 3. Matriz para caracterización y análisis según unidad. Fuente: Elaboración de los autores, según González B, Velosa J, 2016.

\section{Generación de Indicadores de caracterización}

Se procede a la determinación de valores porcentuales de los materiales obtenidos a partir del desarme del equipo en estudio. De manera general los materiales obtenidos corresponden a plásticos, metales ferrosos, metales no ferrosos ( $\mathrm{Al}, \mathrm{Cu}$ ) y componentes electrónicos como la placa de circuito integrado, cables y conectores. En esta instancia se debe declarar los valores porcentuales en relación con el peso total del equipo, para los cual se procede con los siguientes indicadores:

Indicador 1: Índice (\%) en masa por tipo de material

Fórmula: $\frac{m_{\text {mat } 1}}{m_{U}} \times 100$

Donde.

$m_{\text {matt: }}$ masa en gramos material 1

$m_{u}:$ masa del equipo analizado(unidad). 
Indicador 2: Índice (\%) en masa por categoría propuesta

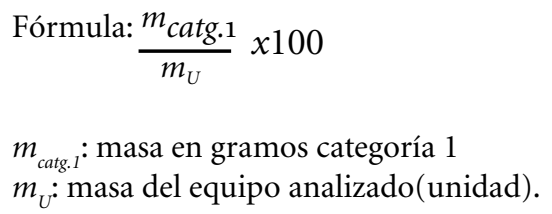

Indicador 3: Índice de Peligrosidad

Se utilizará de referencia la normativa ambiental aplicable a los residuos en Chile, el decreto 148/2004 sobre Reglamento Sanitario sobre Manejo de Residuos Peligrosos (presencia de metales pesados en sus componentes), el cual establece las condiciones sanitarias y de seguridad mínimas a que deberá someterse en general el manejo de residuos, incluyendo operaciones de recuperación y reciclaje (Ver tabla 4).

\begin{tabular}{|c|l|l|l|}
\hline MATERIAL & \multicolumn{1}{|c|}{$\begin{array}{c}\text { CALIFICACIÓN } \\
\text { peligroso/No peligroso }\end{array}$} & $\begin{array}{c}\text { CÓDIGO IDENTIFICACIÓN } \\
\text { n.D.S 148 }\end{array}$ & \multicolumn{1}{|c|}{ DESCRIPCIÓN } \\
\hline Polímeros & No peligroso & B3010 & Residuos sólidos de material plástico \\
\hline Metales & No peligroso & A1180 & $\begin{array}{l}\text { Residuos de metales y de aleaciones de metales, en } \\
\text { forma metálica y no dispensable }\end{array}$ \\
\hline PCB & Peligroso & A1180 & $\begin{array}{l}\text { Montajes eléctricos y electrónicos de desecho 0 } \\
\text { chatarra de éstos que contengan componentes como } \\
\text { por ejemplo, cadmio, mercurios, plomo }\end{array}$ \\
\hline Vidrio CRT & Peligroso & $\begin{array}{l}\text { Montajes eléctricos y electrónicos de desecho 0 } \\
\text { chatarra de éstos que contengan componentes como } \\
\text { vidrios de tubos de rayos catódicos y otros }\end{array}$ \\
\hline Vidrio & No peligroso & B2020 & Residuos de vidrios en forma no dispersable \\
\hline Otros & No aplica & No aplica & $\begin{array}{l}\text { Mezcla de otros materiales no clasificados. Ej.: cables, } \\
\text { conectores, tornillos, pequeñas piezas de polímeros } \\
\text { o metales }\end{array}$ \\
\hline
\end{tabular}

Tabla 4. Peligrosidad de material según Reglamento sanitario sobre manejo de residuos peligrosos 148/20. Fuente: Elaboración de los autores a partir de D.S. 148/2004.

Para el método de cálculo de índice de peligrosidad se requiere las masas parciales de los materiales clasificado como peligroso y la masa total del RAEE en gramos:

Índice de Peligrosidad:

$$
\text { Fórmula: } \frac{m_{\text {respel }}}{m_{U}} \times 100
$$




\section{Donde}

$\mathrm{m}_{\text {respel }}:$ masa total de materiales clasificados como peligrosos

$\mathrm{m}_{\mathrm{U}}$ : masa del equipo analizado (unidad).

Indicador 4: Índice de reciclabilidad

Para el siguiente punto entenderemos la reciclabilidad como la "capacidad de las piezas componentes, materiales o ambos, para ser desviados del flujo del fin de vida para ser reciclados" (UNE 26516 / 2010). A partir de esto se propone la evaluación de la capacidad de reciclaje de los materiales caracterizados a partir de un RAEE. Para realizar este análisis se definieron 2 niveles de reciclabilidad:

- Reciclabilidad factible: corresponde a la posibilidad actual en el país de ingresar estos materiales a la cadena de reciclaje.

- Reciclabilidad potencial: existe tecnología de reciclar estos materiales, pero actualmente no se encuentra disponible en las cadenas de reciclaje en el país.

Estos niveles están propuestos por los autores y representan una situación actual que puede modificarse en el tiempo, lo que debe considerarse para ponderar el indicador en otros estudios. A estos materiales se les asignó esta característica de reciclabilidad factible o potencial, según lo indica la Tabla 5.

\begin{tabular}{|l|l|}
\hline Material & Reciclabilidad \\
\hline Metales & Factible \\
\hline Vidrio & Factible \\
\hline Polímeros & Potencial \\
\hline PCB & Potencial \\
\hline Varios & Potencial \\
\hline
\end{tabular}

Tabla 5. Clasificación de material según capacidad de reciclaje. Fuente: Elaboración de los autores, basado en UNE 26516 / 2010.

Para el método de cálculo de índice de reciclabilidad se requiere las masas parciales de los materiales clasificados por tipo y la masa total del RAEE en gramos. La referencia para el cálculo de este indicador es propuesta a partir de norma UNE 26516 del año 2010, sobre reciclabilidad, recuperabilidad y método de cálculo para vehículo de carretera. A continuación, se presenta un cálculo de índice de reciclabilidad: 
- Índice Reciclabilidad Factible:

Fórmula: $\frac{m_{M+} m_{V}}{m_{U}} \times 100$

- Índice Reciclabilidad Potencial:

Fórmula: $\frac{m_{p}+m_{p c b}+m_{v}}{m_{U}} \times 100$

Donde:

$m_{M}$ : masa de materiales clasificados como metales.

$m_{V}:$ masa de materiales clasificados como vidrio.

$m_{U}:$ masa del equipo analizado(unidad).

$m_{p}$ : masa de materiales clasificados como polímeros.

$m_{p c b}:$ masa de materiales clasificados como pcb, print circuit board.

$m_{v}$ : masa de materiales clasificados como varios.

\section{Estudio de casos}

Se procede a aplicar la metodología señalada en los cinco (5) siguientes equipos AEE para constituir el análisis:

- Torre CPU

- Impresora Multifuncional

- Pantalla CRT

- Impresora de escritorio

- Teclado computador (periférico de entrada)

En el trabajo de laboratorio se registra lo constatado en el proceso de desarme de los equipos señalados para consignar así dicha información en tablas a modo de resumen. Sigue la Tabla 6 a modo de ejemplificación de lo ejecutado. 


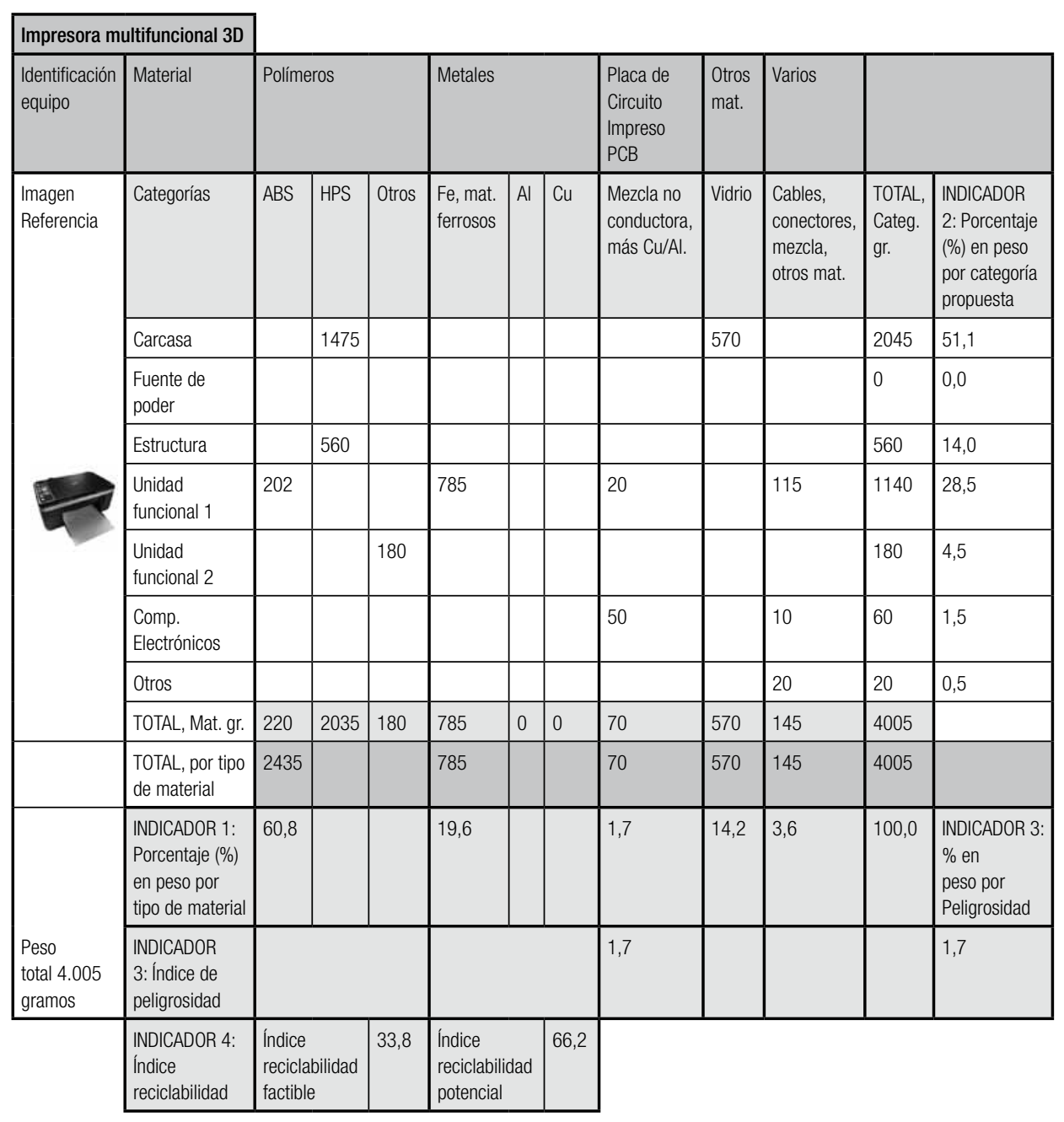

Tabla 6. Constatación y registro de indicadores según $1^{\circ}$ equipo. Fuente: Desarrollo de los autores. 


\section{Resultados y discusión}

De acuerdo a lo verificado en la aplicación del modelo procedimental es factible aseverar lo siguiente:

- Se comprueba la presencia importante de componentes metálicos (férricos y no férricos) pero de acuerdo a lo declarado por diversos autores, éstos ya cuentan con una conocida valorización en el mercado. Lo que permite inferir que su proceso de recuperación es mas frecuente.

Es de interés por tanto, atender a la porción de material constituida por los plásticos y dentro de éstos el ABS en primera prioridad y el HIPS en segunda (Ver Tabla 7). De acuerdo a la revisión del estado del arte existente no hay frecuencia de reciclaje de éstos polímeros, lo que abre un nuevo espacio para el desarrollo de proyectos asociados al manejo y aprovechamiento de estos residuos. Representando por tanto una línea de continuidad a este trabajo, relevando tecnologías que pudieran adaptarse para mejorar el rendimiento y valorización de estos residuos.

- La reciclabilidad potencial (Ver Tabla 8) establecida para 3 de los 5 equipos está asociada directamente con la tipología de material que compone en su mayoría sus subsistemas estructurales (polímeros) Lo que determinaría la posibilidad para la aplicación de nuevos proyectos asociados a nuevas adaptaciones en los procesos tecnológicos existentes para su reciclaje, que en este caso y en razón de la factibilidad inmediata debiera ser del tipo mecánico.(solo las piezas, que no contengan sustancias peligrosas dentro de sus componentes al ser fabricadas)

\begin{tabular}{|l|l|l|l|}
\hline Tipo de Polímero / Equipo & ABS & HIPS & Otros \\
\hline Torre CPU & 11 & & \\
\hline Impr. Multif HP & 5 & 51 & 5 \\
\hline Pantalla CRT & 20 & & 2 \\
\hline Impr. escritorio Epson & 51 & 2 & 6 \\
\hline Teclado & 83 & & 2 \\
\hline
\end{tabular}

Tabla 7. Tipo de polímeros y fracción de masa del total en equipos analizados. Fuente: Desarrollo de los autores, en base a ensayos en laboratorio. 


\begin{tabular}{|c|c|c|c|c|c|c|c|c|c|c|c|c|c|c|}
\hline \multicolumn{6}{|c|}{$\begin{array}{l}\text { INDICADOR 2: Índice (\%) en masa por categoria } \\
\text { propuesta }\end{array}$} & \multicolumn{6}{|c|}{$\begin{array}{l}\text { INDICADOR 1: Índice (\%) en masa por tipo de } \\
\text { material }\end{array}$} & \multirow[t]{2}{*}{$\begin{array}{l}\text { INDICADOR } \\
\text { 3: Índice de } \\
\text { peligrosidad }\end{array}$} & \multicolumn{2}{|c|}{$\begin{array}{l}\text { INDICADOR 4: } \\
\text { Índice de } \\
\text { reciclabilidad }\end{array}$} \\
\hline Otros & $\begin{array}{l}\text { Comp. } \\
\text { Electr }\end{array}$ & $\begin{array}{l}\text { Unidad } \\
\text { Funcional }\end{array}$ & Estructura & $\begin{array}{l}\text { Fte. } \\
\text { Poder }\end{array}$ & Carcasa & $\begin{array}{l}\text { Material } \\
\% \text { del } \\
\text { total / } \\
\text { Equipo }\end{array}$ & Polímeros & Metales & PCB & Vidrio & Otros & & Factible & Potencial \\
\hline 0 & 18 & 14 & 30 & 13 & 26 & $\begin{array}{l}\text { Torre } \\
\text { CPU }\end{array}$ & 11 & 69 & 16 & 0 & 4 & 16 & 69 & 31 \\
\hline 1 & 2 & 33 & 14 & 0 & 51 & $\begin{array}{l}\text { Impr. } \\
\text { Multif HP }\end{array}$ & 61 & 20 & 2 & 14 & 4 & 2 & 34 & 66 \\
\hline 3 & 14 & 63 & 0 & 0 & 20 & $\begin{array}{l}\text { Pantalla } \\
\text { CRT }\end{array}$ & 22 & 4 & 3 & 63 & 9 & 66 & 66 & 34 \\
\hline 9 & 5 & 36 & 5 & 0 & 46 & $\begin{array}{l}\text { Impr. } \\
\text { escritorio }\end{array}$ & 59 & 28 & 4 & 0 & 9 & 4 & 28 & 72 \\
\hline 13 & 4 & 0 & 0 & 0 & 83 & Teclado & 85 & 0 & 2 & 0 & 13 & 2 & 0 & 100 \\
\hline
\end{tabular}

Tabla 8. Matriz resumen caracterización equipos RAEE. Fuente: Desarrollo de los autores.

- La valorización de residuos no solamente está relacionada con indicadores monetarios establecidos por el precio vigente del material a recuperar. También es factible prospectar una nueva forma de valorización, considerando su impacto medioambiental y la urgencia de su recuperación. Lo que explora nuevos intereses para la factibilidad de reciclaje.

- La obtención de datos de caracterización de RAEE, propuesta por este trabajo, permite la individualización de los materiales que componen estos residuos, información necesaria para evaluar las opciones de reciclaje o brechas tecnológicas para su adecuada valorización. Todo esto orientado a mejorar el diseño de los modelos de gestión para su manejo.

- La relevancia del modelo de caracterización propuesta se justifica en razón a que el peso y composición de material de estos residuos pueden ser variables en el tiempo. Tanto por cambios en el comportamiento del consumidor, así como en el concepto del diseño de producto como resultado de la continua evolución tecnológica. Esta situación hace necesario la ejecución de levantamientos sucesivos para la caracterización de estos residuos, que permitan disminuir la incertidumbre respecto a estos datos.

- La metodología propuesta también se puede adaptar para la caracterización de otro tipo de residuos permitiendo levantar datos de interés que en su conjunto pueden aportar a optimizar las cadenas de reciclaje para los residuos.

- Desde el punto de vista del Diseño del Producto, es relevante analizar los componentes y materiales de una determinada unidad, esto entendido como una orientación hacia su 
ecodiseño, concepto por lo demás ya incluido en la Ley REP. En la experiencia comparada con otros países, ya existen normativas que exigen al fabricante un mínimo de capacidad de reciclabilidad de los productos que ponen en el mercado y esto declarado un indicador.

El presente trabajo espera ser un avance en estas metodologías que en un mediano plazo deberían integrarse a los marcos normativos relacionados con la gestión de residuos en nuestro país.

\section{Referencias}

Baldé, C. P.; Forti, V.; Gray, V.; Kuehr, R. \& Stegmann, P. (2017). The global E-waste Monitor. Quantities, flows and resources. Bonn/Geneva/Vienna. International Telecommunication Union (ITU) \& International Solid Waste Association (ISWA).

Conde Ortiz, M. (1992). Manual para la Identificación de plásticos. (Tesis de pregrado, Instituto politécnico Nacional D.F, México).

Fajardo, D. (2017). ¿Qué falta para tener una ley de reciclaje 100\% operativa? Pulso, La Tercera. Recuperado de http://www.pulso.cl/hub-sustenibilidad/falta-una-ley-reciclaje100-operativa/.

Fernández Promastro, F. (2013). Minería urbana y la gestión de los residuos electrónicos. Mendoza, Argentina: Editorial Isalud.

Habib, K.; Parajuly, K. \& Wenzel, H. (2015). Tracking the flow of resources in electronic waste-the case of end-of-life computer hard disk drives. Environment science \& techno$\log y, 49$ (20), 12441-12449.

Heinz, B.; Silva, U. y Ott, D. (2009). Reciclaje de residuos electrónicos en América latina: panorama general, desafíos y potencial. Santiago, Chile: SUR Corporación de Estudios Sociales y Educación.

MacArthur, E. (2015). Growth within: A circular economy vision for a competitive Europe. Isle of Wight, Inglaterra: Foundation and McKinsey Center.

McDonough, W. \& Braungart M. (2005). Cradle to Cradle. Madrid: Ed.Farrar, Straus and Giroux (BYR).

Ministerio de Salud (2004). Reglamento sanitario sobre manejo de residuos peligrosos. D.S. 148/. 2004. Santiago, Chile: Autor.

Ministerio del Medio Ambiente (2016). Ley REP 20920: Responsabilidad extendida del productor. Santiago, Chile: Autor. https://tesis.ipn.mx/bitstream/handle/123456789/17123/ 25-1-11813.pdf?sequence $=1$ \&isAllowed $=\mathrm{y}$.

Ministerio del Medio Ambiente (2009). Diagnostico producción, importación y distribución de productos electrónicos y manejo de los equipos fuera de uso. Santiago, Chile: Autor.

Park, Y. J. \& Fray, D. J. (2009). Recovery of high purity precious metals from printed circuit boards. Journal of Hazardous Materials, 164 (2-3), 1152-1158.

UNE (2010) Norma Española UNE 26516: 2010 Vehículos de carretera reciclabilidad, recuperabilidad y método de cálculo.

UNESCO (2012). Education for Sustainable Development Sourcebook. The United Nations Educational. Francia: UNESCO. 
Velosa, J.; González, G. y Rolón, B. (2016). Caracterización y estudio del reciclaje de residuos de aparatos eléctricos y electrónicos (RAEE). (Vol3, No2). México: Universidad de Guanajuato.

Zeng, X.; Yang, C.; Chiang, J. F. \& Li, J. (2017). Innovating e-waste management: From macroscopic to microscopic scales. Science of the Total Environment, 575, 1-5.

\begin{abstract}
The following work arises from the survey of information that allowed to evaluate the current situation of electrical and electronic waste (WEEE) in Chile. Identifying the characterization methods in each material to establish conditions, appraisals and procedures in the proper handling of these in post of the new Law of Extended Producer Responsibility (EPR). The result obtained establishes a procedural model applicable to the Teaching of Engineering and Design among other disciplines, which will systematize decision making and planning from an ecoefficient perspective, thus promoting projects of technological and economic interest for sustainable management of electrical and electronic waste.
\end{abstract}

Keywords: Law EPR - Electrical and Electronic Waste - Waste Management - Method for characterization.

Resumo: O trabalho surge a partir do relevamento de informação que permitiu avaliar a situação atual dos resíduos elétricos e eletrônicos (RAEE) em Chile. Identificando os métodos de caracterização em cada material para estabelecer condições, valorizações e procedimentos na gestão adequada de estes em favor da nova Lei de Responsabilidade estendidas do Produtor (REP). O resultado obtido estabelece um modelo procedimental aplicável à engenharia e o design entre outras disciplinas, que permitirá sistematizar a tomada de decisões e o planejamento desde uma perspectiva eco - eficiente, potenciando deste modo projetos de interesse tecnológico e econômico para o manejo sustentável dos resíduos elétricos e eletrônicos.

Palavras chave: Lei REP - Resíduos elétricos e eletrônicos - Gestão de resíduos - Método para a caracterização. 